\title{
Article \\ Asymptotic Stability of Solutions to a Class of Second-Order Delay Differential Equations
}

\author{
Gennadii V. Demidenko*(D) and Inessa I. Matveeva \\ Laboratory of Differential and Difference Equations, Sobolev Institute of Mathematics, 4, Acad. Koptyug Avenue, \\ 630090 Novosibirsk, Russia; matveeva@math.nsc.ru \\ * Correspondence: demidenk@math.nsc.ru
}

check for updates

Citation: Demidenko, G.V.; Matveeva, I.I. Asymptotic Stability of Solutions to a Class of Second-Order Delay Differential Equations.

Mathematics 2021, 9, 1847. https: / / doi.org/10.3390/math9161847

Received: 7 July 2021

Accepted: 30 July 2021

Published: 5 August 2021

Publisher's Note: MDPI stays neutral with regard to jurisdictional claims in published maps and institutional affiliations.

Copyright: (c) 2021 by the authors. Licensee MDPI, Basel, Switzerland. This article is an open access article distributed under the terms and conditions of the Creative Commons Attribution (CC BY) license (https:/ / creativecommons.org/licenses/by/ $4.0 /)$.
Abstract: We consider a class of second-order nonlinear delay differential equations with periodic coefficients in linear terms. We obtain conditions under which the zero solution is asymptotically stable. Estimates for attraction sets and decay rates of solutions at infinity are established. This class of equations includes the equation of vibrations of the inverted pendulum, the suspension point of which performs arbitrary periodic oscillations along the vertical line.

Keywords: delay differential equations; periodic coefficients; inverted pendulum; asymptotic stability; Lyapunov differential equation

\section{Introduction}

We consider the following second-order delay differential equation:

$$
y^{\prime \prime}(\xi)+\alpha y^{\prime}(\xi)+(\beta+\omega \varphi(\omega \xi)) \psi(y(\xi))+\gamma y(\xi-\tau)+\sigma y^{\prime}(\xi-\tau)=0, \quad \xi>0,
$$

where $\alpha>0, \beta<0, \omega>0, \gamma, \sigma$ are parameters, $\varphi(s)$ is a continuous $T$-periodic function such that the following equality holds:

$$
\int_{0}^{T} \varphi(s) d s=0,
$$

$\psi(y)$ is a smooth real-valued vector-function, $\psi(0)=0$, and $\tau>0$ is a delay. Our aims are to obtain the conditions for the asymptotic stability of the zero solution to (1), to establish estimates for attraction sets of the zero solution and estimates for decay rates of solutions to $(1)$ as $t \rightarrow \infty$.

In the case $\gamma=\sigma=0$ (1) coincides with the equation of the "inverted pendulum", $(\varphi(t)=\sin t, \psi(y)=\sin y)$, with the oscillating suspension point along the vertical line according to the law $x(t)=a \sin (\omega t)[1,2]$. In the literature, this pendulum is often called the Kapitsa pendulum. (1) describes oscillations of a "controlled inverted pendulum", the suspension point of which performs arbitrary periodic oscillations.

It should be noted that a large number of works are devoted to the study of inverted pendulums with vibrating suspension points, pendulums on carts, systems consisting of several pendulums, with control, etc. (for example, refer to [3,4] and the bibliography therein). One of the main problems is the stability problem for these mechanical systems. Many papers are devoted to finding stability conditions, but very few papers contain estimates characterizing stabilization rates to equilibrium positions.

The present paper continues a cycle of authors' research [5-11] on the stability of solutions to ordinary differential equations and delay equations with periodic coefficients in linear terms. In [5], the following systems of linear differential equations were considered:

$$
\frac{d x}{d t}=A(t) x, \quad t>0,
$$


where $A(t)$ is an $n \times n$ matrix with continuous $T$-periodic entries. The following criterion for the asymptotic stability of the zero solution to (3) was established in terms of solvability of a special boundary value problem for the Lyapunov differential equation

$$
\frac{d}{d t} H+H A(t)+A^{*}(t) H=-C(t) .
$$

Theorem 1 ([5]). The zero solution to (3) is asymptotically stable if and only if there is a Hermitian solution to the boundary value problem:

$$
\begin{aligned}
& \frac{d}{d t} H+H A(t)+A^{*}(t) H=-C(t), \quad 0 \leq t \leq T, \\
& H(0)=H(T)>0,
\end{aligned}
$$

where the Hermitian matrix $C(t)$ with continuous entries on $[0, T]$ is positive definite.

Here and further, $S>0(S<0)$ denotes that $S$ is a positive (negative) definite Hermitian matrix. We use the spectral matrix norm everywhere. Note that differential equations of the form (4) have been considered in many papers when studying qualitative behavior of solutions to differential equations with variable coefficients (for example, see [12-17]).

Using the solution $H(t)$ to the boundary value problem (5), we can point out the radius of the disk containing all the multipliers of (3) and obtain estimates characterizing the decay rate of solutions to (3) at infinity [5].

Theorem 2 ([5]). If the zero solution to (3) is asymptotically stable, then the following estimate holds for its solutions:

$$
\|x(t)\| \leq \sqrt{\|H(0)\|\left\|H^{-1}(t)\right\| \|}\|x(0)\| \exp \left(-\int_{0}^{t} \frac{c_{1}(s)}{2\|H(s)\|} d s\right), \quad t>0,
$$

where $H(t)$ is a T-periodic extension of the solution to (5) on the semi-axis $\{t \geq 0\}, c_{1}(t)>0$ is the minimal eigenvalue of the matrix $C(t)$.

Remark 1. Estimate (6) coincides with Krein's estimate for systems of differential equations with constant coefficients (for instance, refer to [13]).

Remark 2. Theorems 1 and 2 allow us to study the asymptotic stability of the zero solution to (3) without finding multipliers.

Using the results mentioned above, the authors investigated the asymptotic stability of the zero solution to the following nonlinear systems [6]:

$$
\frac{d x}{d t}=A(t) x+f(t, x), \quad t>0,
$$

where $f(t, x)$ is a smooth real-valued vector-function, $f(t, 0)=0$. Using the solution $H(t)$ to the boundary value problem (5), estimates for attraction sets of the zero solution were obtained and estimates of exponential decrease of solutions to (7) at infinity were established.

On the basis of these studies, introducing special Lyapunov-Krasovskii functionals, the authors investigated the stability of solutions to some classes of delay differential equations with periodic coefficients in linear terms (for example, see [7-11]). Conditions for the asymptotic stability of the zero solution and estimates characterizing decay rates of solutions as $t \rightarrow \infty$ were obtained in these papers. These estimates are analogs of Krein's estimate. 
We will use some assertions that follow from [7,8,11]. Consider the following system of delay differential equations:

$$
\frac{d}{d t} x(t)=A(t) x(t)+B(t) x(t-\tau)+F(t, x(t), x(t-\tau)), \quad t>0,
$$

where $A(t), B(t)$ are $n \times n$ matrices with continuous real-valued T-periodic entries, $\tau>0$ is a delay and $F(t, u, v)$ is a continuous real-valued vector-function on $[0, \infty) \times \mathbb{R}^{n} \times \mathbb{R}^{n}$. We assume that $F(t, u, v)$ satisfies the Lipschitz condition with respect to $u$ on every compact set $G \subset[0, \infty) \times \mathbb{R}^{n} \times \mathbb{R}^{n}$ and the following estimate:

$$
\|F(t, u, v)\| \leq p\|u\|^{1+\delta}, \quad t \geq 0, \quad u, v \in \mathbb{R}^{n}
$$

for some constants $p \geq 0, \delta>0$.

At first we formulate our results for the linear systems.

Theorem 3 ([7]). Let $F(t, u, v) \equiv 0$. Suppose that there are matrices

$$
H(t)=H^{*}(t) \in C^{1}[0, T] \text { and } K(s)=K^{*}(s) \in C^{1}[0, \tau]
$$

such that

$$
H(0)=H(T)>0, \quad K(s)>0, \quad \frac{d}{d s} K(s)<0, \quad s \in[0, \tau],
$$

and the following differential inequality

$$
\frac{d}{d t} H(t)+H(t) A(t)+A^{*}(t) H(t)+H(t) B(t) K^{-1}(\tau) B^{*}(t) H(t)<-K(0), \quad t \in[0, T],
$$

holds. Then the zero solution to (8) is asymptotically stable.

We introduce the following matrix:

$$
P(t)=-\left[\frac{d}{d t} H(t)+H(t) A(t)+A^{*}(t) H(t)\right]-H(t) B(t) K^{-1}(\tau) B^{*}(t) H(t)-K(0) .
$$

If the conditions of Theorem 3 hold, then $P(t)>0$. Denote by $p_{1}(t)>0$ the minimal eigenvalue of the matrix $P(t)$ and by $k>0$ the maximal number such that the following inequality holds:

$$
\frac{d}{d s} K(s)+k K(s) \leq 0, \quad s \in[0, \tau] .
$$

Consider the initial value problem for (8):

$$
\begin{aligned}
& \frac{d}{d t} x(t)=A(t) x(t)+B(t) x(t-\tau)+F(t, x(t), x(t-\tau)), \quad t>0, \\
& x(t)=x_{0}(t), \quad t \in[-\tau, 0], \quad x(+0)=x_{0}(0),
\end{aligned}
$$

where $x_{0}(t) \in C([-\tau, 0])$ is a given vector-function.

Theorem $4([7,8])$. Let $F(t, u, v) \equiv 0$. Suppose that there are matrices $H(t)$ and $K(s)$ satisfying the conditions of Theorem 3. Then the following estimate holds for the solution to (12):

$$
\|x(t)\| \leq \sqrt{\frac{V\left(0, x_{0}\right)}{h_{1}(t)}} \exp \left(-\int_{0}^{t} \frac{\varepsilon(\xi)}{2\|H(\xi)\|} d \xi\right), \quad t>0,
$$

where $h_{1}(t)>0$ is the minimal eigenvalue of the matrix $H(t)$.

$$
\varepsilon(t)=\min \left\{p_{1}(t), k\|H(t)\|\right\}
$$




$$
V\left(0, x_{0}\right)=\left\langle H(0) x_{0}(0), x_{0}(0)\right\rangle+\int_{-\tau}^{0}\left\langle K(-s) x_{0}(s), x_{0}(s)\right\rangle d s
$$

Inequality (13) is an analog of Krein's estimate. Consider nonlinear systems of the form (8). Supposing that the conditions of Theorem 3 hold, we define $R$ as follows:

$$
\begin{gathered}
R^{\delta / 2}=\left(1-\exp \left(-\frac{\delta}{2} \int_{0}^{T} \frac{\varepsilon(s)}{\|H(s)\|} d s\right)\right) \\
\times\left(p \delta \int_{0}^{T} \frac{\|H(\xi)\|}{h_{1}^{1+\delta / 2}(\xi)} \exp \left(-\frac{\delta}{2} \int_{0}^{\xi} \frac{\varepsilon(s)}{\|H(s)\|} d s\right) d \xi\right)^{-1} .
\end{gathered}
$$

Theorem 5 ([7,11]). Let the conditions of Theorem 3 hold. Then, the zero solution to (8) is asymptotically stable and the set of real-valued functions

$$
\mathcal{E}=\left\{x_{0}(t) \in C[-\tau, 0]: V\left(0, x_{0}\right)<R\right\}
$$

is an attraction set of the zero solution. Moreover, the solution to the initial value problem (12) with $x_{0}(t) \in \mathcal{E}$ satisfies the following estimate:

$$
\|x(t)\| \leq \sqrt{\frac{V\left(0, x_{0}\right)}{h_{1}(t)}} \exp \left(-\int_{0}^{t} \frac{\varepsilon(\xi)}{2\|H(\xi)\|} d \xi\right)\left(1-R^{-\delta / 2}\left(V\left(0, x_{0}\right)\right)^{\delta / 2}\right)^{-1 / \delta}, \quad t>0
$$

Using the results mentioned above, auxiliary transforms are performed and preliminary assertions are proved in Section 2. The main results are formulated and proved in Section 3.

\section{Auxiliary Results}

Consider the following linear equation:

$$
y^{\prime \prime}(\xi)+\alpha y^{\prime}(\xi)+(\beta+\omega \varphi(\omega \xi)) y(\xi)+\gamma y(\xi-\tau)+\sigma y^{\prime}(\xi-\tau)=0, \quad \xi>0,
$$

where the function $\varphi(s)$ satisfies (2). In the next section we prove that the zero solution to (14) is asymptotically stable under certain conditions on the function $\varphi(s)$ and parameters $\alpha, \beta, \gamma, \sigma$, and $\omega$. In order to prove this result, we need to perform a number of transforms.

Using the following change of variables:

$$
t=\omega \xi, \quad \widetilde{y}(t)=y\left(\frac{t}{\omega}\right), \quad \mu=\frac{1}{\omega}, \quad \widetilde{\tau}=\frac{\tau}{\mu},
$$

we rewrite (14) as follows:

$$
\widetilde{y}^{\prime \prime}(t)+\alpha \mu \widetilde{y}^{\prime}(t)+\left(\beta \mu^{2}+\mu \varphi(t)\right) \widetilde{y}(t)+\gamma \mu^{2} \widetilde{y}(t-\widetilde{\tau})+\sigma \mu \widetilde{y}^{\prime}(t-\widetilde{\tau})=0 .
$$

We introduce the vector-function:

$$
v(t)=\left(\begin{array}{c}
v_{1}(t) \\
v_{2}(t)
\end{array}\right)=\left(\begin{array}{c}
\widetilde{y}(t) \\
\widetilde{y}^{\prime}(t)
\end{array}\right)
$$

This equation is equivalent to the system of linear delay differential equations:

$$
\frac{d v(t)}{d t}=A(t, \mu) v(t)+B(\mu) v(t-\widetilde{\tau})
$$


where

$$
A(t, \mu)=\left(\begin{array}{cc}
0 & 1 \\
-\beta \mu^{2}-\mu \varphi(t) & -\alpha \mu
\end{array}\right), \quad B(\mu)=\left(\begin{array}{cc}
0 & 0 \\
-\gamma \mu^{2} & -\sigma \mu
\end{array}\right) .
$$

We introduce the following functions:

$$
v_{1}(t)=(1+\mu a(t)) u_{1}(t), \quad v_{2}(t)=\mu b(t) u_{1}(t)+\mu c(t) u_{2}(t) .
$$

Then, (15) is reduced to the following system:

$$
\frac{d u(t)}{d t}=\widehat{A}(t, \mu) u(t)+\widehat{B}(t, \mu) u(t-\widetilde{\tau}),
$$

where the entries of $\widehat{A}(t, \mu)$ have the following form:

$$
\begin{gathered}
\widehat{a}_{11}(t)=\frac{\mu\left(b(t)-a^{\prime}(t)\right)}{1+\mu a(t)}, \quad \widehat{a}_{12}(t)=\frac{\mu c(t)}{1+\mu a(t)}, \\
\widehat{a}_{21}(t)=-\frac{\mu}{c(t)}\left(\beta+\alpha b(t)+\mu \beta a(t)+\varphi(t) a(t)+\frac{b(t)\left(b(t)-a^{\prime}(t)\right)}{1+\mu a(t)}\right)-\frac{b^{\prime}(t)+\varphi(t)}{c(t)}, \\
\widehat{a}_{22}(t)=-\mu\left(\alpha+\frac{b(t)}{1+\mu a(t)}\right)-\frac{c^{\prime}(t)}{c(t)}, \\
\widehat{B}(t, \mu)=-\frac{\mu}{c(t)}\left(\begin{array}{cc}
0 \\
\gamma(1+\mu a(t-\widetilde{\tau}))+\sigma b(t-\widetilde{\tau}) & \sigma c(t-\widetilde{\tau})
\end{array}\right) .
\end{gathered}
$$

We show below that there are $T$-periodic functions $a(t), b(t), c(t)$ such that the vectorfunction $u(t)=\left(\begin{array}{l}u_{1}(t) \\ u_{2}(t)\end{array}\right)$ satisfies the following system:

$$
\frac{d u(t)}{d t}=\mu \widetilde{A}(t, \mu) u(t)+\mu \widetilde{B}(t, \mu) u(t-\widetilde{\tau}) .
$$

By analogy with [6], we take $c(t) \equiv 1$ and choose the functions $a(t), b(t)$ such that the following equalities hold:

$$
a^{\prime}(t)-b(t)=0, \quad b^{\prime}(t)+\varphi(t)=0 .
$$

Hence, we have (17), where

$$
\begin{gathered}
\widetilde{A}(t, \mu)=\left(\begin{array}{cc}
0 & \frac{1}{1+\mu a(t)} \\
-\beta-\alpha b(t)-\mu \beta a(t)-\varphi(t) a(t) & -\alpha-\frac{b(t)}{1+\mu a(t)}
\end{array}\right), \\
\widetilde{B}(t, \mu)=\left(\begin{array}{cc}
0 & 0 \\
-\gamma(1+\mu a(t-\widetilde{\tau}))-\sigma b(t-\widetilde{\tau}) & -\sigma
\end{array}\right) .
\end{gathered}
$$

Using the following representation:

$$
\frac{1}{1+\mu a(t)}=1-\mu a(t)+\frac{\mu^{2} a^{2}(t)}{1+\mu a(t)}
$$

we rewrite $\widetilde{A}(t, \mu)$ as follows:

$$
\widetilde{A}(t, \mu)=\widetilde{A}_{1}+\widetilde{A}_{2}(t, \mu)+\mu \widetilde{A}_{3}(t, \mu),
$$


where

$$
\begin{gathered}
\widetilde{A}_{1}=\left(\begin{array}{cc}
0 & 1 \\
-\beta-\frac{1}{T} \int_{0}^{T} \varphi(\zeta) a(\zeta) d \zeta & -\alpha
\end{array}\right) \\
\widetilde{A}_{2}(t, \mu)=\left(\begin{array}{cc}
0 & -\mu a(t) \\
-\alpha b(t)-\mu \beta a(t)-\varphi(t) a(t)+\frac{1}{T} \int_{0}^{T} \varphi(\zeta) a(\zeta) d \zeta & (\mu a(t)-1) b(t)
\end{array}\right), \\
\widetilde{A}_{3}(t, \mu)=\left(\begin{array}{cc}
0 & \frac{a^{2}(t)}{1+\mu a(t)} \\
0 & -\frac{a^{2}(t) b(t)}{1+\mu a(t)}
\end{array}\right) .
\end{gathered}
$$

We show below that there are the functions $a(t), b(t)$ satisfying (18), such that the spectrum of the matrix $\widetilde{A}_{1}$ belongs to the left half-plane, the matrix $\widetilde{A}_{2}(t, \mu)$ is T-periodic, and the following equality holds:

$$
\int_{0}^{T} \widetilde{A}_{2}(\zeta, \mu) d \zeta=0
$$

Taking into account (18), we seek the functions $a(t), b(t)$ in the following form:

$$
\begin{gathered}
b(t)=-\int_{t_{1}}^{t} \varphi(s) d s, \\
a(t)=-\int_{t_{2}}^{t} \int_{t_{1}}^{s} \varphi(\xi) d \xi d s .
\end{gathered}
$$

Clearly, $t_{1}, t_{2} \in[0, T]$ must be such that the following equalities hold:

$$
\int_{0}^{T} a(\zeta) d \zeta=0, \quad \int_{0}^{T} b(\zeta) d \zeta=0
$$

At first we construct the function $b(t)$. Obviously, by the mean value theorem, there exists $t_{1} \in[0, T]$ such that the following equality holds:

$$
\int_{0}^{T} \zeta \varphi(\zeta) d \zeta=T \int_{t_{1}}^{T} \varphi(\zeta) d \zeta
$$

Taking into account the following equalities:

$$
\int_{0}^{T} \zeta \varphi(\zeta) d \zeta=\int_{0}^{T} \int_{\zeta}^{T} \varphi(s) d s d \zeta, \quad T \int_{t_{1}}^{T} \varphi(s) d s=\int_{0}^{T} \int_{t_{1}}^{T} \varphi(s) d s d \zeta
$$

we can rewrite (25) as follows:

$$
\int_{0}^{T} \int_{t_{1}}^{\zeta} \varphi(s) d s d \zeta=0
$$


Take the function $b(t)$ in the form (23) with $t_{1}$ defined above. By (2), the function $b(t)$ is $T$-periodic; moreover, from (26), we have

$$
\int_{0}^{T} b(\zeta) d \zeta=0
$$

We now construct the function $a(t)$. At first, we show that there exists $t_{2} \in[0, T]$ such that the relation holds:

$$
\frac{1}{2} \int_{0}^{T} \zeta^{2} \varphi(\zeta) d \zeta+T \int_{t_{2}}^{T} \int_{t_{1}}^{s} \varphi(\zeta) d \zeta d s=\frac{T^{2}}{2} \int_{t_{1}}^{T} \varphi(\zeta) d \zeta .
$$

Indeed, we can represent the right-hand side in the following form:

$$
\frac{T^{2}}{2} \int_{t_{1}}^{T} \varphi(\zeta) d \zeta=\int_{0}^{T} s \int_{s}^{T} \varphi(\zeta) d \zeta d s+\int_{0}^{T} s \int_{t_{1}}^{s} \varphi(\zeta) d \zeta d s .
$$

Obviously, for the first summand, the equality is valid:

$$
\int_{0}^{T} s \int_{s}^{T} \varphi(\zeta) d \zeta d s=\frac{1}{2} \int_{0}^{T} \zeta^{2} \varphi(\zeta) d \zeta
$$

By the mean value theorem, the second summand can be written as follows:

$$
\int_{0}^{T} s \int_{t_{1}}^{s} \varphi(\zeta) d \zeta d s=T \int_{t_{2}}^{T} \int_{t_{1}}^{s} \varphi(\zeta) d \zeta d s,
$$

where $t_{2} \in[0, T]$. Hence we obtain (27).

We show that (27) can be rewritten in the following form:

$$
\int_{0}^{T} \int_{t_{2}}^{\zeta} \int_{t_{1}}^{s} \varphi(\xi) d \xi d s d \zeta=0
$$

Indeed, integrating by parts, we have

$$
\begin{gathered}
\int_{0}^{T} \int_{t_{2}}^{\zeta} \int_{t_{1}}^{s} \varphi(\xi) d \zeta d s d \zeta=T \int_{t_{2}}^{T} \int_{t_{1}}^{s} \varphi(\zeta) d \zeta d s-\int_{0}^{T} s \int_{t_{1}}^{s} \varphi(\zeta) d \zeta d s \\
\quad=T \int_{t_{2}}^{T} \int_{t_{1}}^{s} \varphi(\zeta) d \zeta d s-\frac{T^{2}}{2} \int_{t_{1}}^{T} \varphi(\zeta) d \zeta+\frac{1}{2} \int_{0}^{T} \zeta^{2} \varphi(\zeta) d \zeta .
\end{gathered}
$$

Consequently, (27) is equivalent to (28). Therefore, the function $a(t)$ can be taken in the form (24) with $t_{2}$ defined above.

We now show that $a(t)$ is $T$-periodic. Indeed, according to the definition, we have the following identities:

$$
\frac{d}{d t}[a(t+T)-a(t)] \equiv b(t+T)-b(t) \equiv 0
$$


On the other hand, by the properties of the function $b(t)$, we have

$$
a(T)-a(0)=\int_{0}^{T} b(s) d s=0 .
$$

Hence, $a(t+T) \equiv a(t)$.

Thus, we have specified the functions $a(t)$ and $b(t)$ satisfying (18) such that the matrix $\widetilde{A}_{2}(t, \mu)$ is $T$-periodic and the following equality holds:

$$
\int_{0}^{T} \widetilde{A}_{2}(\zeta, \mu) d \zeta=0
$$

Lemma 1. The spectrum of the matrix $\widetilde{A}_{1}$ belongs to the left half-plane if and only if $\alpha>0$ and

$$
\frac{1}{T} \int_{0}^{T}\left(\int_{0}^{\zeta} \varphi(s) d s\right)^{2} d \zeta>\left(\frac{1}{T} \int_{0}^{T} \zeta \varphi(\zeta) d \zeta\right)^{2}-\beta
$$

Proof. From the explicit form (20) of the matrix $\widetilde{A}_{1}$, it follows that its spectrum belongs to the left half-plane if and only if $\alpha>0$ and

$$
\beta+\frac{1}{T} \int_{0}^{T} \varphi(\zeta) a(\zeta) d \zeta>0
$$

We show below that this inequality is equivalent to (30). To perform this, we make a series of transforms of the integral in (31). Integrating by parts and taking into account (2), we have

$$
\begin{aligned}
& \int_{0}^{T} \varphi(\zeta) a(\zeta) d \zeta=\left.\int_{0}^{\zeta} \varphi(\xi) d \xi a(\zeta)\right|_{0} ^{T}-\int_{0}^{T} \int_{0}^{\zeta} \varphi(\xi) d \xi a^{\prime}(\zeta) d \zeta \\
& =-\left.\int_{0}^{\zeta} \int_{0}^{s} \varphi(\xi) d \xi d s a^{\prime}(\zeta)\right|_{0} ^{T}+\int_{0}^{T} \int_{0}^{\zeta} \int_{0}^{s} \varphi(\xi) d \xi d s a^{\prime \prime}(\zeta) d \zeta \\
& =-\int_{0}^{T} \int_{0}^{s} \varphi(\xi) d \xi d s a^{\prime}(T)+\int_{0}^{T} \int_{0}^{\zeta} \int_{0}^{s} \varphi(\xi) d \xi d s a^{\prime \prime}(\zeta) d \zeta .
\end{aligned}
$$

By (24), this equality can be written as follows:

$$
\int_{0}^{T} \varphi(\zeta) a(\zeta) d \zeta=\int_{0}^{T} \int_{0}^{s} \varphi(\xi) d \xi d s \int_{t_{1}}^{T} \varphi(\zeta) d \zeta-\int_{0}^{T} \int_{0}^{\zeta} \int_{0}^{s} \varphi(\xi) d \xi d s \varphi(\zeta) d \zeta .
$$

Using (25), we obtain

$$
\begin{gathered}
\int_{0}^{T} \varphi(\zeta) a(\zeta) d \zeta=\frac{1}{T} \int_{0}^{T} \int_{0}^{s} \varphi(\xi) d \xi d s \int_{0}^{T} \zeta \varphi(\zeta) d \zeta-\int_{0}^{T} \int_{0}^{\zeta} \int_{0}^{s} \varphi(\xi) d \xi d s \varphi(\zeta) d \zeta \\
=\frac{1}{T} \int_{0}^{T}(T-s) \varphi(s) d s \int_{0}^{T} \zeta \varphi(\zeta) d \zeta
\end{gathered}
$$




$$
-\left.\int_{0}^{\zeta} \int_{0}^{s} \varphi(\xi) d \xi d s \int_{0}^{\zeta} \varphi(\eta) d \eta\right|_{0} ^{T}+\int_{0}^{T}\left(\int_{0}^{\zeta} \varphi(\xi) d \xi\right)\left(\int_{0}^{\zeta} \varphi(\eta) d \eta\right) d \zeta .
$$

Taking into account (2), we have

$$
\int_{0}^{T} \varphi(\zeta) a(\zeta) d \zeta=-\frac{1}{T}\left(\int_{0}^{T} \zeta \varphi(\zeta) d \zeta\right)^{2}+\int_{0}^{T}\left(\int_{0}^{\zeta} \varphi(\xi) d \zeta\right)^{2} d \zeta
$$

The equivalence of (30) and (31) follows immediately from this formula.

The lemma is proved.

\section{Main Results}

At first we consider (14). We introduce the following notation:

$$
\widetilde{\beta}=\left(\frac{1}{T} \int_{0}^{T} \zeta \varphi(\zeta) d \zeta\right)^{2}-\frac{1}{T} \int_{0}^{T}\left(\int_{0}^{\zeta} \varphi(s) d s\right)^{2} d \zeta-\beta
$$

Taking into account the transforms performed in the previous section, the matrix $\widetilde{A}_{1}$ given in (20) has the following form:

$$
\widetilde{A}_{1}=\left(\begin{array}{cc}
0 & 1 \\
\widetilde{\beta} & -\alpha
\end{array}\right)
$$

If $\alpha>0, \widetilde{\beta}<0$, then its spectrum belongs to the left half-plane. In this case the following Lyapunov equation:

$$
H_{1} \widetilde{A}_{1}+\widetilde{A}_{1}^{*} H_{1}=-I
$$

has a unique solution $H_{1}=H_{1}^{*}>0$; moreover, it can be written as follows (for example, see [13]):

$$
H_{1}=\int_{0}^{\infty} e^{t \widetilde{A}_{1}^{*}} e^{t \widetilde{A}_{1}} d t
$$

We establish below some conditions for the coefficients of (14) under which it is possible to guarantee the asymptotic stability of the zero solution to (14).

Theorem 6. If $\alpha>0, \widetilde{\beta}<0$ and

$$
\sqrt{\sigma^{2}+\left(\gamma\left(1+\frac{T^{2}}{2 \omega} \max _{t \in[0, T]}|\varphi(t)|\right)+\sigma T \max _{t \in[0, T]}|\varphi(t)|\right)^{2}} \leq \frac{q}{4\left\|H_{1}\right\|}, \quad 0<q<1,
$$

then, the zero solution to (14) is asymptotically stable for sufficiently small $\mu>0$.

Proof. We will use the transforms performed in the previous section. According to the notation, $\mu=\frac{1}{\omega}$. We assume that $0<\mu<\left(\frac{T^{2}}{2} \max _{t \in[0, T]}|\varphi(t)|\right)^{-1}$. By (24), this condition guarantees that $1+\mu a(t)>0$.

Taking into account these transforms, in order to prove the theorem, it is sufficient to establish the asymptotic stability of the zero solution to the linear system of delay differential equations:

$$
\frac{d u(t)}{d t}=\mu \widetilde{A}(t, \mu) u(t)+\mu \widetilde{B}(t, \mu) u(t-\widetilde{\tau}),
$$


where

$$
\widetilde{A}(t, \mu)=\widetilde{A}_{1}+\widetilde{A}_{2}(t, \mu)+\mu \widetilde{A}_{3}(t, \mu),
$$

the matrices $\widetilde{A}_{2}(t, \mu), \widetilde{A}_{3}(t, \mu)$, and $\widetilde{B}(t, \mu)$ are defined in (19), (21), and (22), respectively. According to Theorem 3 , if there are matrices $H(t, \mu)$ and $K(s)$ satisfying (10) and (11) and such that the following matrix inequality holds:

$$
\begin{gathered}
-\left[\frac{d}{d t} H(t, \mu)+\mu H(t, \mu)\left(\widetilde{A}_{1}+\widetilde{A}_{2}(t, \mu)+\mu \widetilde{A}_{3}(t, \mu)\right)+\mu\left(\widetilde{A}_{1}+\widetilde{A}_{2}(t, \mu)+\mu \widetilde{A}_{3}(t, \mu)\right)^{*} H(t, \mu)\right] \\
-K(0)>\mu^{2} H(t, \mu) \widetilde{B}(t, \mu) K^{-1}(\tau) \widetilde{B}^{*}(t, \mu) H(t, \mu),
\end{gathered}
$$

then the zero solution to (32) is asymptotically stable.

It is not difficult to calculate the spectral norm of the matrix $\widetilde{B}(t, \mu)$ given in (19); moreover,

$$
\|\widetilde{B}(t, \mu)\|=\sqrt{(\gamma(1+\mu a(t-\widetilde{\tau}))+\sigma b(t-\widetilde{\tau}))^{2}+\sigma^{2}} .
$$

Using (23) and (24), we have the following estimates:

$$
\begin{gathered}
\|\widetilde{B}(t, \mu)\| \leq \sqrt{\left(\gamma\left(1+\mu \max _{t \in[0, T]}|a(t)|\right)+\sigma \max _{t \in[0, T]}|b(t)|\right)^{2}+\sigma^{2}} \\
\leq \sqrt{\sigma^{2}+\left(\gamma\left(1+\frac{\mu T^{2}}{2} \max _{t \in[0, T]}|\varphi(t)|\right)+\sigma T \max _{t \in[0, T]}|\varphi(t)|\right)^{2}} .
\end{gathered}
$$

Hence, by the conditions of the theorem, we obtain

$$
\|\widetilde{B}(t, \mu)\| \leq \frac{q}{4\left\|H_{1}\right\|^{\prime}}, \quad 0<q<1 .
$$

We take $k>0$ such that $\rho=q e^{k \tilde{\tau} / 2}<1$.

Consider the following matrix:

$$
H(t, \mu)=\frac{1}{\mu} H_{1}-H_{2}(t, \mu),
$$

where $H_{1}$ is the above solution to the Lyapunov equation,

$$
H_{2}(t, \mu)=H_{1} \int_{0}^{t} \widetilde{A}_{2}(\eta, \mu) d \eta+\int_{0}^{t} \widetilde{A}_{2}^{*}(\eta, \mu) d \eta H_{1}
$$

Obviously, $H(0, \mu)=\frac{1}{\mu} H_{1}>0$ and, by (29), we have $H(T, \mu)=H(0, \mu)$. Clearly,

$$
\begin{gathered}
\quad \frac{d}{d t} H(t, \mu)+\mu H(t, \mu)\left(\widetilde{A}_{1}+\widetilde{A}_{2}(t, \mu)+\mu \widetilde{A}_{3}(t, \mu)\right) \\
+\mu\left(\widetilde{A}_{1}+\widetilde{A}_{2}(t, \mu)+\mu \widetilde{A}_{3}(t, \mu)\right)^{*} H(t, \mu)=-C(t, \mu),
\end{gathered}
$$

where

$$
\begin{gathered}
C(t, \mu)=I+\mu\left[H_{2}(t, \mu)\left(\widetilde{A}_{1}+\widetilde{A}_{2}(t, \mu)\right)+\left(\widetilde{A}_{1}+\widetilde{A}_{2}(t, \mu)\right)^{*} H_{2}(t, \mu)\right] \\
-\mu^{2}\left[H(t, \mu) \widetilde{A}_{3}(t, \mu)+\widetilde{A}_{3}^{*}(t, \mu) H(t, \mu)\right] .
\end{gathered}
$$

By construction, there is $\mu_{1}, 0<\mu_{1}<\left(\frac{T^{2}}{2} \max _{t \in[0, T]}|\varphi(t)|\right)^{-1}$, such that the following inequality holds:

$$
\|H(t, \mu)\| \leq \frac{2}{\mu}\left\|H_{1}\right\|, \quad t \in[0, T],
$$

for $0<\mu \leq \mu_{1}$. Then there exists $\mu_{0}, 0<\mu_{0} \leq \mu_{1}$, such that the following estimate is valid:

$$
C(t, \mu)>\rho I, \quad t \in[0, T],
$$


for $\mu \leq \mu_{0}$. Hence, the matrix $H(t, \mu)$ is a Hermitian solution to the problem of the form (5). Consequently, as shown in [5],

$$
H(t, \mu)>0, \quad t \in[0, T] .
$$

We take the following matrix:

$$
K(s)=\frac{\rho}{2} e^{-k s} I .
$$

Clearly, the matrices $H(t, \mu)$ and $K(s)$ satisfy (10) and (11). We now show that (33) is valid for $\mu<\mu_{0}$. From (34), (36) we have

$$
\begin{gathered}
-\left[\frac{d}{d t} H(t, \mu)+\mu H(t, \mu)\left(\widetilde{A}_{1}+\widetilde{A}_{2}(t, \mu)+\mu \widetilde{A}_{3}(t, \mu)\right)+\mu\left(\widetilde{A}_{1}+\widetilde{A}_{2}(t, \mu)+\mu \widetilde{A}_{3}(t, \mu)\right)^{*} H(t, \mu)\right] \\
-K(0)>\rho I-K(0)=\frac{\rho}{2} I .
\end{gathered}
$$

Hence, it remains to show that the following inequality holds:

$$
\mu^{2} H(t, \mu) \widetilde{B}(t, \mu) K^{-1}(\tau) \widetilde{B}^{*}(t, \mu) H(t, \mu) \leq \frac{\rho}{2} I, \quad t \in[0, T],
$$

for $\mu<\mu_{0}$. Indeed, by (35), we obtain

$$
\begin{gathered}
\mu^{2}\left\|H(t, \mu) \widetilde{B}(t, \mu) K^{-1}(\tau) \widetilde{B}^{*}(t, \mu) H(t, \mu)\right\| \leq \frac{2 \mu^{2} e^{k \widetilde{\tau}}}{\rho}\|H(t, \mu)\|^{2}\|\widetilde{B}(t, \mu)\|^{2} \\
\leq \frac{8 e^{k \widetilde{\tau}}}{\rho}\left\|H_{1}\right\|^{2}\|\widetilde{B}(t, \mu)\|^{2} \leq \frac{q^{2} e^{k \widetilde{\tau}}}{2 \rho}=\frac{\rho}{2}
\end{gathered}
$$

In other words, (37) holds.

The theorem is proved.

Example 1. Let

$$
\varphi(t)=\sum_{k=1}^{l}\left(a_{k} \cos (k t)+b_{k} \sin (k t)\right) .
$$

In this case, the condition $\widetilde{\beta}<0$ has the following form:

$$
\beta>-\frac{1}{2} \sum_{k=1}^{l}\left(\left(\frac{a_{k}}{k}\right)^{2}+\left(\frac{b_{k}}{k}\right)^{2}\right) .
$$

Using Theorem 4, we have estimates characterizing decay rates of solutions to (32) at infinity. Making the inverse change of variables, we obtain asymptotic estimates for solutions to (15) and for solutions to (14).

We now consider nonlinear Equation (1). Suppose that the function $\psi(y)$ satisfies the following estimate:

$$
\left|\psi(y)-\psi^{\prime}(0) y\right| \leq \check{p}|y|^{1+\delta}, \quad \check{p} \geq 0, \quad \delta>0,
$$

and $\psi^{\prime}(0) \neq 0$. Rewrite $(1)$ as follows:

$$
\begin{gathered}
y^{\prime \prime}(\xi)+\alpha y^{\prime}(\xi)+(\beta+\omega \varphi(\omega \xi)) \psi^{\prime}(0) y(\xi) \\
+(\beta+\omega \varphi(\omega \xi))\left(\psi(y(\xi))-\psi^{\prime}(0) y(\xi)\right)+\gamma y(\xi-\tau)+\sigma y^{\prime}(\xi-\tau)=0, \quad \xi>0 .
\end{gathered}
$$

Consider the following linear equation:

$$
y^{\prime \prime}(\xi)+\alpha y^{\prime}(\xi)+(\beta+\omega \varphi(\omega \xi)) \psi^{\prime}(0) y(\xi)+\gamma y(\xi-\tau)+\sigma y^{\prime}(\xi-\tau)=0 .
$$


Introducing the notation $\check{\beta}=\beta \psi^{\prime}(0)$ and $\breve{\omega}=\omega \psi^{\prime}(0)$, we obtain an equation of the form (14), for solutions to which the assertions proved above are valid. Using the following change of variables:

$$
t=\breve{\omega} \xi, \quad \check{y}(t)=y\left(\frac{t}{\check{\omega}}\right), \quad \check{\mu}=\frac{1}{\breve{\omega}^{\prime}}, \quad \check{\tau}=\frac{\tau}{\check{\mu}^{\prime}}
$$

we rewrite (39) as follows:

$$
\begin{gathered}
\check{y}^{\prime \prime}(t)+\alpha \check{\mu} \check{y}^{\prime}(t)+\left(\check{\beta} \check{\mu}^{2}+\check{\mu} \varphi(t)\right) \check{y}(t)+\gamma \check{\mu}^{2} \check{y}(t-\check{\tau})+\sigma \check{\mu} \check{y}^{\prime}(t-\check{\tau}) \\
+\left(\check{\beta} \check{\mu}^{2}+\check{\mu} \varphi(t)\right)\left(\psi(\check{y}(t))-\psi^{\prime}(0) \check{y}(t)\right)=0 .
\end{gathered}
$$

Introduce the following vector-function:

$$
\check{v}(t)=\left(\begin{array}{c}
\check{v}_{1}(t) \\
\check{v}_{2}(t)
\end{array}\right)=\left(\begin{array}{c}
\check{y}(t) \\
\check{y}^{\prime}(t)
\end{array}\right) .
$$

By (39), we have the nonlinear system of delay differential equations:

$$
\frac{d \check{v}(t)}{d t}=\check{A}(t, \check{u}) \check{v}(t)+\check{B}(\check{\mu}) v(t-\check{\tau})+F(t, \check{v}(t), \check{\mu}),
$$

where

$$
\begin{gathered}
\check{A}(t, \check{\mu})=\left(\begin{array}{cc}
0 & 1 \\
-\check{\beta} \breve{u}^{2}-\check{\mu} \varphi(t) & -\alpha \check{\mu}
\end{array}\right), \quad \check{B}(\check{\mu})=\left(\begin{array}{cc}
0 & 0 \\
-\gamma \check{\mu}^{2} & -\sigma \breve{\mu}
\end{array}\right), \\
F(t, \check{v}(t), \check{\mu})=\left(\begin{array}{c}
0 \\
-\left(\check{\beta} \breve{\mu}^{2}+\check{\mu} \varphi(t)\right)\left(\psi\left(\check{v}_{1}(t)\right)-\psi^{\prime}(0) \check{v}_{1}(t)\right)
\end{array}\right) .
\end{gathered}
$$

From (38) we obtain the following estimate:

$$
\|F(t, \check{v}, \check{u})\| \leq p\|\check{v}\|^{1+\delta}, \quad p=\left(|\check{\beta}| \breve{\mu}^{2}+\check{\mu} \max _{t \in[0, T]}|\varphi(t)|\right) \check{p} .
$$

Hence, (42) is included in the class of systems of the form (8) with the right-hand side satisfying (9). Then, by Theorem 5, we obtain an attraction set for the zero solution to (1) and the estimates of the exponential decrease of solutions to (1) with the initial data from this attraction set.

Theorem 7. If $\alpha>0$,

$$
\frac{1}{T} \int_{0}^{T}\left(\int_{0}^{\zeta} \varphi(s) d s\right)^{2} d \zeta>\left(\frac{1}{T} \int_{0}^{T} \zeta \varphi(\zeta) d \zeta\right)^{2}-\beta,
$$

and

$$
\sqrt{\sigma^{2}+\left(\gamma\left(1+\frac{T^{2}}{2 \omega} \max _{t \in[0, T]}|\varphi(t)|\right)+\sigma T \max _{t \in[0, T]}|\varphi(t)|\right)^{2}} \leq \frac{q}{4\left\|H_{1}\right\|^{\prime}}, \quad 0<q<1,
$$

then the zero solution to (1) is asymptotically stable for sufficiently small $\mu>0$.

Remark 3. As was mentioned above, in the case $\gamma=\sigma=0$, (1) coincides with the equation of the "inverted pendulum" $(\varphi(t)=\sin t, \psi(y)=\sin y)$ with the oscillating suspension point along the vertical line by according to the law $x(t)=a \sin (\omega t)[1,2]$. The conditions of the asymptotic stability of the form (43) coincide with known N.N. Bogolyubov's conditions obtained by the averaging method (for instance, refer to $[18,19])$. 
Author Contributions: Conceptualization, G.V.D. and I.I.M.; methodology, G.V.D. and I.I.M.; validation, G.V.D. and I.I.M.; investigation, G.V.D. and I.I.M.; writing一original draft preparation, G.V.D. and I.I.M.; writing-review and editing, G.V.D. and I.I.M.; supervision, G.V.D. and I.I.M. Both authors have read and agreed to the published version of the manuscript.

Funding: The authors are supported by the Russian Foundation for Basic Research (project No. 1829-10086).

Institutional Review Board Statement: Not applicable.

Informed Consent Statement: Not applicable.

Data Availability Statement: Not applicable.

Acknowledgments: The authors thank the anonymous reviewers for their helpful remarks.

Conflicts of Interest: The authors declare no conflict of interest.

\section{References}

1. Bogolyubov, N.N. Perturbation theory in nonlinear mechanics. Sb. Tr. Inst. Stroitel'n. Mekhaniki Akad. Nauk Ukrain. SSR 1950, 14, 9-34.

2. Kapitsa, L.P. Dynamical stability of a pendulum with vibrating suspension point. Zh. Eksper. Teoret. Fiz. 1951, $21,588-597$.

3. Blekhman, I.I. Vibration Mechanics; Fizmatlit: Moscow, Russia, 1994.

4. Boubaker, O.; Iriarte, R. (Eds.) The Inverted Pendulum in Control Theory and Robotics: From Theory to New Innovations; The Institution of Engineering and Technology: London, UK, 2017.

5. Demidenko, G.V.; Matveeva, I.I. On stability of solutions to linear systems with periodic coefficients. Sib. Math. J. 2001, 42, 282-296. [CrossRef]

6. Demidenko, G.V.; Matveeva, I.I. On stability of solutions to quasilinear periodical systems of differential equations. Sib. Math. J. 2004, 45, 1041-1052. [CrossRef]

7. Demidenko, G.V.; Matveeva, I.I. Stability of solutions of differential equations with retarded argument and periodic coefficients in the linear terms. Sib. Math. J. 2007, 48, 824-836. [CrossRef]

8. Demidenko, G.V.; Matveeva, I.I. On estimates of solutions to systems of differential equations of neutral type with periodic coefficients. Sib. Math. J. 2014, 55, 866-881. [CrossRef]

9. Demidenko, G.V.; Matveeva, I.I. Estimates for solutions to a class of time-delay systems of neutral type with periodic coefficients and several delays. Electron. J. Qual. Theory Differ. Equ. 2015, 2015, 1-22. [CrossRef]

10. Matveeva, I.I. On the exponential stability of solutions of periodic systems of the neutral type with several delays. Differ. Equ. 2017, 53, 725-735. [CrossRef]

11. Demidenko, G.V.; Matveeva, I.I.; Skvortsova, M.A. Estimates for the solutions of neutral type differential equations with periodic coefficients in the linear terms. Sib. Math. J. 2019, 60, 828-841. [CrossRef]

12. Malkin, I.G. A Theory of Stability of Motion; Nauka: Moscow, Russia, 1966.

13. Daleckii, J.L.; Krein, M.G. Stability of Solutions of Differential Equations in Banach Space; Translations of Mathematical Monographs; American Mathematical Society: Providence, RI, USA, 1974; Volume 43.

14. Yakubovich, V.A.; Starzhinskii, V.M. Linear Differential Equations with Periodic Coefficients and Their Applications; Nauka: Moscow, Russia, 1972.

15. Andreev, Y.N. Control of Finite-Dimensional Linear Objects; Nauka: Moscow, Russia, 1976.

16. Rozenvasser, E.N. Lyapunov Exponents in the Theory of Nonlinear Control Systems; Nauka: Moscow, Russia, 1977.

17. Bodunov, N.A.; Kotchenko, F.F. Dependence of the stability of linear periodic systems on the period. Differentsial'nye Uravneniya 1988, 24, 338-341.

18. Bogolyubov, N.N.; Mitropol'skii, Y.A. Asymptotic Methods in the Theory on Nonlinear Oscillations; Fizmatgiz: Moscow, Russia, 1963.

19. Mitropol'skii, Y.A. The Averaging Method in Nonlinear Mechanics; Naukova Dumka: Kiev, Russia, 1971. 